\title{
A PROBABILISTIC APPROACH TO A BOUNDARY LAYER PROBLEM
}

BY

\author{
WALTER VASILAKY
}

\begin{abstract}
An elliptic second order linear operator is approximated by the transition operator of a Markov chain, and the solution to the corresponding approximate boundary value problem is expanded in terms of a small parameter, up to the first order term. In characterizing the boundary values of the first order term in the expansion, a problem of a boundary layer arises, which is treated by probabilistic methods.
\end{abstract}

1. Introduction. Consider the boundary value problem:

$$
\begin{aligned}
L u(x) \equiv & \sum_{i j=1}^{n} a_{i j}(x) \frac{\partial^{2}}{\partial x_{i} \partial x_{j}} u(x) \\
& +\sum_{j=1}^{n} b_{j}(x) \frac{\partial}{\partial x_{j}} u(x)=-g(x) \\
& \quad \text { for } x \in G=\{x: \phi(x)<0\}, x \in R^{n},
\end{aligned}
$$

and

$$
u(x)=b(x) \text { for } x \in \partial G=\{x: \phi(x)=0\},
$$

where $\phi(x)=0$ is the boundary of a closed, bounded, connected domain $G$ such that $\nabla \phi(x) \neq 0$ for all $x \in \partial G$. Assume that $a_{i j}(x), b_{j}(x), b(x), g(x)$, $\phi(x)$ are in $C_{b}^{\infty}$ and that the matrix $\left(a_{i j}(x)\right)^{n \times m}$ is uniformly positive definite. Let $p_{h}(x, y)$ be a transition density of a Markov chain for which the jumps are uniformly bounded by $c h^{1 / 2}$, where $c$ is a fixed positive constant and $h$ a small parameter. We define a transition operator $\Pi_{h}$ on $C_{b}$ to be

$$
\left(\Pi_{h} f\right)(x)=\int p_{h}\left(x, x^{\prime}\right) f\left(x^{\prime}\right) d x^{\prime} .
$$

Assume that

$$
\lim _{h \rightarrow 0} \frac{\left(\Pi_{h} f\right)(x)-f(x)}{h}=L f(x)
$$

uniformly on compacts of $R^{n}$ for each $f \in C_{b}^{2}$. We formulate the approximate problem of (1.1) to be

Received by the editors September 1, 1976.

AMS (MOS) subject classifications (1970). Primary 60J99; Secondary 65N05.

- American Mathematical Society 1978 


$$
\begin{aligned}
\left(\Pi_{h} u_{h}\right)(x)-u_{h}(x) & =-h g(x), & & x \in \bar{G}, \\
u_{h}(x) & =F(x), & & x \in \bar{G}^{\prime},
\end{aligned}
$$

where $F(x)$ is a continuous extension of the boundary values $b(x)$ into $\bar{G}^{\prime}=\{x: \phi(x)>0\}$. From the probabilistic point of view the solution to (1.3) is

$$
u_{h}(x)=E_{x}^{h} F\left(x_{\tau_{G}}^{h}\right)+h E_{x}^{h}\left(\sum_{j=0}^{\tau_{G}-1} g\left(x_{j}^{h}\right)\right)
$$

where $\tau_{G}$ is the first exit time from $\bar{G}$ of a Markov chain for which (1.2) holds. We obtain an asymptotic expansion of the form

$$
u_{h}(x)=u(x)+\sqrt{h} u_{1}(x)+o(\sqrt{h})
$$

where $u(x)$ is the solution to (1.1) and $u_{1}(x)$ is the solution to another boundary value problem, namely:

$$
L u_{1}(x)=-L_{1} u(x), \quad x \in G ;
$$

where $L_{1}$ is a differential operator determined by the Markov chain, and $u_{1}(x)=b_{1}(x)$ for $x \in \partial G$, where $b_{1}$ is to be determined. To see the kind of difficulties one can anticipate in characterizing $b_{1}(x)$, suppose we postulate that

$$
\left(\Pi_{h} f\right)(x)=\left(I+h L+h^{3 / 2} L_{1}\right)(f)(x)+o\left(h^{3 / 2}\right),
$$

$f \in C_{b}^{\infty}$; then the approximate equation (1.3) can be written in the form

$$
\left(L+h^{1 / 2} L_{1}\right) u_{h}(x)=-g(x)+o\left(h^{1 / 2}\right) .
$$

Thus (1.3) can be viewed as an equation involving a singular perturbation of the operator $L$.

As an example of how the operator $L_{1}$ is determined by the Markov chain, consider a probability distribution $F(y)$ with zero mean, variance 1 , third moment $m_{3} \neq 0$ and density $f(x)$; then for $a(x)>\delta>0, b(x)$ in $C^{\infty}$

$$
\frac{1}{\sqrt{h a(x)}} F\left(\frac{y-x-h b(x)}{\sqrt{h a(x)}}\right)
$$

is a distribution with mean $x+h b(x)$ and variance 1 . We make a change in variable, $y=\sqrt{h a(x)} z+x+h b(x)$ and define $\Pi_{h}$ as

$$
\Pi_{h} u_{h}(x, y)=\int u_{h}(x, x+b(x) h+\sqrt{a(x) h} z) f(z) d z .
$$

Now expanding formally by a Taylor expansion $u_{h}(x, y)$ with respect to $y$ at $y=x$ and collecting terms in powers of $\sqrt{h}$, we obtain: 


$$
\begin{aligned}
\Pi_{h} u_{h}(x, y) & \\
= & {\left[I+h\left(\frac{a(x)}{2} \frac{\partial^{2}}{\partial y^{2}}+b(x) \frac{\partial}{\partial y}\right)+h^{3 / 2}\left(\frac{m_{3}}{3 !}(a(x))^{3 / 2} \frac{\partial^{3}}{\partial y^{3}}\right)\right] u_{h}(x, y) } \\
& +o\left(h^{3 / 2}\right) .
\end{aligned}
$$

Thus

$$
L_{1}=\frac{m_{3}}{3 !}(a(x))^{3 / 2} \frac{\partial^{3}}{\partial y^{3}} .
$$

We know from singular perturbation theory that, in characterizing $b_{1}(x)$, we can anticipate a boundary layer problem. Employing probability methods, we treat the boundary layer problem and obtain the characterization of $b_{1}(x)$.

2. The asymptotic expansion. Since no complications arise in generalizing our results from dimension $n=2$ to $n>2$, we shall limit our discussion to the two dimensional case. It is known that the $\lim _{h \rightarrow 0} u_{h}(x)=u(x)$ for $x \in G$, see [4]. We begin the characterization of the first order term by proving the following:

LEMMA 1. If

$$
\lim _{h \rightarrow 0} \frac{u_{h}(x)-u(x)}{h^{1 / 2}}=u_{1}(x)
$$

exists uniformly on compacts in $G$, then it is of the form $u_{1}(x)=\bar{u}_{1}(x)+\hat{u}_{1}(x)$, where $\hat{u}_{1}(x)$ is the solution to $L \hat{u}_{1}(x)=-L_{1} u(x)$ with zero boundary conditions, and $\bar{u}_{1}(x)$ is harmonic for $L$ inside $G$.

Proof. Let $U(x)$ be a smooth extension of $u(x)$ into $\bar{G}^{\prime}$, i.e., let the inward derivatives of $u(x)$ agree with the corresponding outward derivatives of $U(x)$ on $\partial G$. We note that $u(x)$ possesses these inward derivatives since it is known that if the data are smooth then so is the solution in the case of elliptic boundary value problem [1]. It is easy to check that

$$
U\left(x_{n}^{h}\right)-\sum_{j=0}^{\tau_{G}-1}\left(\Pi_{h}-I\right) U\left(x_{j}^{h}\right)
$$

is a martingale, where $x, x_{1}^{h}, x_{2}^{h}, x_{n}^{h}, \ldots$ is a Markov chain satisfying the assumptions of $\S 1$. Substituting

$$
\left(\Pi_{h}-I\right) U(x)=-h g(x)-h^{3 / 2} g_{1}(x)+o\left(h^{3 / 2}\right),
$$

where $L_{1} u(x)=g_{1}(x)$, in the martingale and taking into account that the expectation of a martingale is the same for all parameter values, that the expectation of the martingale at $t=0$ is $U(x)$ and Doob's stopping theorem, we have 


$$
\begin{aligned}
E_{x}^{h}\left(U\left(x_{\tau_{G}}^{h}\right)-\sum_{j=0}^{\tau_{G}-1}\left(\Pi_{h}-I\right) U\left(x_{\tau_{G}}^{h}\right)\right) \\
=E_{x}^{h} U\left(x_{\tau_{G}}^{h}\right)+h E_{x}^{h} \sum_{j=0}^{\tau_{G}-1} g\left(x_{n}^{h}\right) \\
+h^{3 / 2} E_{x}^{h} \sum_{j=0}^{\tau_{G}-1} g_{1}\left(x_{j}^{h}\right)+E_{x}^{h}\left(\tau_{G}\right) o\left(h^{3 / 2}\right) \\
=U(x) .
\end{aligned}
$$

Since $E_{x}^{h}\left(\tau_{G}\right)<$ const $/ h$ for a discrete time Markov chain, see [2],

$\frac{u_{h}(x)-U(x)}{\sqrt{h}}=\frac{1}{\sqrt{h}}\left(E_{x}^{h}\left(F\left(x_{\tau_{G}}^{h}\right)-U\left(x_{\tau_{G}}^{h}\right)\right)\right)-h E_{x}^{h} \sum_{j=0}^{\tau_{G}-1} g_{1}\left(x_{j}^{h}\right)+o(1)$.

But

$$
\lim _{h \rightarrow 0} h E_{x}^{h}\left(\sum_{j=0}^{\tau_{G}-1} g_{1}\left(x_{j}^{h}\right)\right)=E_{x} \int_{0}^{\tau_{G}} g_{1}\left(x_{t}\right) d t,
$$

where $x_{t}$ is a diffusion process generated by the operator $L$. This limit (let us denote it by $\hat{u}_{1}(x)$ ) is of course the solution to the Poisson equation $L \hat{u}_{1}(x)=$ $-g_{1}(x)$ with zero boundary conditions. Now let

$$
\bar{u}_{1}^{h}(x)=\frac{1}{h^{1 / 2}} E_{x}^{h}\left(F\left(x_{\tau_{G}}^{h}\right)-U\left(x_{\tau_{G}}^{h}\right)\right)
$$

By assumption $\lim _{h \rightarrow 0} \bar{u}_{1}^{h}(x)=\bar{u}_{1}(x)$ exists for $x \in G$. Let $D$ be a compact subset of $G$. Then by the strong Markov property

$$
\bar{u}_{1}^{h}(x)=E_{x}^{h} \bar{u}_{1}^{h}\left(x_{\tau_{D}}^{h}\right)=\int \bar{u}_{1}^{h}(x) H_{h}^{D}(x, d y),
$$

where $\tau_{D}$ is the first exit time from $D$ and $H_{h}^{D}(x, d y)$ is the exit distribution from $D$. Since the $\lim _{h \rightarrow 0} H_{h}^{D}(x, d y)$ is the harmonic measure on $\partial D$, it follows that $\bar{u}_{1}(x)$ is harmonic in $D$. This completes the proof of Lemma 1 .

It remains to prove that the hypothesis of Lemma 1 is satisfied and to obtain the boundary values of $\bar{u}_{1}(x)$. We begin by proving

LEMMA 2.

$$
\lim _{\substack{h \rightarrow 0 \\ x \rightarrow b \in \partial G}}\left|\bar{u}_{1}^{h}(x)-W(b) \frac{1}{\sqrt{h}} E_{x}^{h}\left(\phi\left(x_{\tau_{G}}^{h}\right)\right)\right|=0
$$

where

$$
W(x)=\frac{\langle\nabla(F(x)-U(x)), \nabla \phi(x)\rangle}{\|\nabla \phi(x)\|^{2}}
$$

$(\langle\cdot, \cdot\rangle$ denotes inner-product $)$. 
Proof. Let $V(x)=F(x)-U(x)$; then

$$
\bar{u}_{1}^{h}(x)=\left(1 / h^{1 / 2}\right) E_{x}^{h} V\left(x_{\tau_{G}}^{h}\right) ;
$$

if we expand $V(x)$ in the normal direction to $\phi(x)=0$, we obtain

$$
V(x)=\frac{\langle V(x), \nabla \phi(x)\rangle}{\|\nabla \phi(x)\|^{2}} \phi(x)+O\left(\phi^{2}(x)\right) .
$$

Since the jumps of the Markov chain are uniformly bounded by $c h^{1 / 2}$, we have $O\left(\phi^{2}\left(x_{\tau_{G}}^{h}\right)\right)=O(h)$. Thus

$$
\bar{u}_{1}^{h}(x)=\frac{1}{\sqrt{h}} E_{x}^{h}\left(W\left(x_{\tau_{G}}^{h}\right) \phi\left(x_{\tau_{G}}^{h}\right)\right)+O\left(h^{1 / 2}\right) .
$$

Now for $b$ on $\partial G$

$$
\begin{aligned}
\lim _{\substack{h \rightarrow 0 \\
x \rightarrow b}} & \frac{1}{\sqrt{h}}\left|E_{x}^{h}\left(W\left(x_{\tau_{G}}^{h}\right) \phi\left(x_{\tau_{G}}^{h}\right)\right)-W(b) E_{x}^{h} \phi\left(x_{\tau_{G}}^{h}\right)\right| \\
& =\lim _{\substack{h \rightarrow 0 \\
x \rightarrow b}} \frac{1}{\sqrt{h}}\left|E_{x}^{h}\left[\phi\left(x_{\tau_{G}}^{h}\right)\left(W\left(x_{\tau_{G}}^{h}\right)-W(b)\right)\right]\right| \\
& \leqslant \lim _{\substack{h \rightarrow 0 \\
x \rightarrow b}} k E_{x}^{h}\left|W\left(x_{\tau_{G}}^{h}\right)-W(b)\right|=0 .
\end{aligned}
$$

The last inequality follows from the assumption that the jumps of the Markov chain are uniformly bounded by $c h^{1 / 2}$. Assuming the continuity of $W(x)$, the last equality follows from the fact that $V_{n}(x)=E_{x}^{h}\left[\left|W\left(x_{\tau_{G}}^{h}\right)-W(b)\right|\right]$ converges uniformly in $x \in \bar{G}$ to the solution of $L V=0$ with $V=\mid W(\cdot)-$ $W(b) \mid$ on $\partial G$. This completes the proof of Lemma 2.

Thus, by Lemma 2 , to identify the boundary value of $\bar{u}_{1}(x)$ at $b$, we must identify

$$
\lim _{x \rightarrow b} \lim _{h \rightarrow 0} \frac{1}{h^{1 / 2}} E_{x}^{h} \phi\left(x_{\tau_{G}}^{h}\right) .
$$

To this end we require several preliminary lemmas.

Lemma 3. Assume the transition density $p_{h}(x, y)$ is such that

$$
\lim _{\substack{h \rightarrow 0 \\ x \rightarrow b}} h p_{h}\left(x, x+h^{1 / 2} z\right)=p_{0}^{b}(z)
$$

where $p_{0}^{b}(z)$ is a bivariate probability density. Then for $f$ in $C_{b}^{\infty}$

$$
\lim _{\substack{h \rightarrow 0 \\ x \rightarrow b}} \iint_{R^{2}} \frac{f(\phi(y)-\phi(x))}{\sqrt{h}} p_{h}(x, y) d y=\int_{R} f(s) p^{b}(s) d s,
$$

where $p^{b}(s)$ is the marginal density of $\langle\nabla \phi(b), Z\rangle$ and $Z$ is a random vector with density $p_{0}^{b}(z)$. 
Proof. By a Taylor expansion

$$
\phi(y)=\phi(x)+\langle\nabla \phi(x),(y-x)\rangle+O\left(\|y-x\|^{2}\right) .
$$

Thus

$$
\begin{aligned}
\iint & f\left(\frac{\phi(y)-\phi(x)}{\sqrt{h}}\right) p_{h}(x, y) d y \\
\quad & \iint f\left(\frac{\langle\nabla \phi(x),(y-x)\rangle+O\left(\|y-x\|^{2}\right)}{\sqrt{h}}\right) p_{h}(x, y) d y \\
& =\iint\left(f\left(\frac{1}{\sqrt{h}}\langle\nabla \phi(x),(y-x)\rangle\right)+\frac{1}{\sqrt{h}} O\left(\|y-x\|^{2}\right)\right) p_{h}(x, y) d y
\end{aligned}
$$

where $O\left(\|y-x\|^{2}\right) \leqslant k\|y-x\|^{2}$. But assumption (1.2) implies

$$
\lim _{h \rightarrow 0} \frac{1}{h} \iint\|y-x\|^{2} p_{h}(x, y) d y=\operatorname{trace}\left(a_{i j}(x)\right) \text {; }
$$

thus

$$
\lim _{h \rightarrow 0} \frac{1}{\sqrt{h}} \iint\|y-x\|^{2} p_{h}(x, y) d y=0 .
$$

Taking $z=(y-x) / \sqrt{h}$ we have

$$
\begin{aligned}
\iint_{R^{2}} f( & \left.\frac{1}{\sqrt{h}}\langle\nabla \phi(x),(y-x)\rangle\right) p_{h}(x, y) d y \\
\quad & \iint_{R^{2}} f(\langle\nabla \phi(x), z\rangle) h p_{h}(x, x+\sqrt{h z}) d z .
\end{aligned}
$$

But

$$
\lim _{\substack{h \rightarrow 0 \\ x \rightarrow b}} h p_{h}(x, x+\sqrt{h} z)=p_{0}^{b}(z)
$$

Therefore,

$$
\lim _{\substack{h \rightarrow 0 \\ x \rightarrow b}} \iint_{R^{2}} f(\langle\nabla \phi(x), z\rangle) h p_{h}(x, x+\sqrt{h} z) d z=\int_{R} f(s) p^{b}(s) d s .
$$

This completes the proof of Lemma 3.

LEMMA 4. Let

$$
z_{h}(n)=(1 / \sqrt{h}) \phi\left(x_{n}^{h}\right),
$$

where $\left\{x_{n}^{h}\right\}$ is a Markov chain starting at $x \in G$ with transition density $p_{h}(x, y)$. Then for every $k, z_{h}(1)-z_{h}(0), z_{h}(2)-z_{h}(1), \ldots, z_{h}(k)-z_{h}(k-1)$ converge weakly to $z(1)-z(0), z(2)-z(1), \ldots, z(k)-z(k-1)$ as $h \rightarrow 0$ and $x \rightarrow b$ where $z(n)(n=0,1,2, \ldots)$ is a random walk with transition density $p^{b}(s)$. 
Proof. To show that any finite number of increments of $z_{h}(n)(n=0,1$, $2, \ldots)$ converge weakly to the corresponding finite number of increments of $z(n)(n=0,1,2, \ldots)$, it suffices to show that for any $k$,

$$
\begin{array}{r}
\lim _{\substack{h \rightarrow 0 \\
x \rightarrow b}} E_{x}^{h}\left(f_{1}\left(z_{h}(1)-z_{h}(0)\right) f_{2}\left(z_{h}(2)-z_{h}(1)\right) \cdots f_{k}\left(z_{h}(k)-z_{h}(k-1)\right)\right) \\
=E_{b}\left(f_{1}(z(1)-z(0)) f_{2}(z(2)-z(1)) \cdots f_{k}(z(k)-z(k-1))\right)
\end{array}
$$

where $f_{j}(j=1,2, \ldots k)$ are any continuous, bounded functions. Now

$$
\begin{aligned}
& E_{x}^{h} f_{1}\left(z_{h}(1)-z_{h}(0)\right) f_{2}\left(z_{h}(2)-z_{h}(1)\right) \cdots f_{k}\left(z_{h}(k)-z_{h}(k-1)\right) \\
& =\int_{R^{2 k}} f_{1}\left(\frac{\phi\left(x_{1}\right)-\phi(x)}{\sqrt{h}}\right) f_{2}\left(\frac{\phi\left(x_{2}\right)-\phi\left(x_{1}\right)}{\sqrt{h}}\right) \cdots f_{k}\left(\frac{\phi\left(x_{k}\right)-\phi\left(x_{k-1}\right)}{\sqrt{h}}\right) \\
& \quad \times p_{h}\left(x, x_{1}\right) p_{h}\left(x_{1}, x_{2}\right) \cdots p_{h}\left(x_{k-1}, x_{k}\right) d x_{1} d x_{2} \cdots d x_{k} .
\end{aligned}
$$

We shall evaluate one factor at a time; in the limit this will permit us to write the above expectation as a product of expectations. Evaluating the limit of the $k$ th factor first, i.e., by Lemma 3

$$
\lim _{\substack{h \rightarrow 0 \\ x_{k-1} \rightarrow b}} \iint_{R^{2}} f_{k}\left(\frac{\phi\left(x_{k}\right)-\phi\left(x_{k-1}\right)}{\sqrt{h}}\right) d x_{k}=\int_{R} f(s) p^{b}(s) d s, \quad x_{k} \in R^{2} .
$$

Similarly, by Lemma 3 we evaluate the limit of the $(k-1)$ th factor, and again obtain $\int_{R} f(s) p^{b}(s) d s$. Continuing to evaluate each consecutive factor in the same manner, we obtain

$$
\begin{array}{r}
\lim _{\substack{h \rightarrow 0 \\
x_{i} \rightarrow b \\
i=k-1, k-2, \ldots, 0}} E_{x}^{h} f_{1}\left(z_{h}(1)-z_{h}(0)\right) \cdots f_{k}\left(z_{h}(k)-z_{h}(k-1)\right) \\
=\int_{R} f_{1}(s) p^{b}(s) d s \cdots \int_{R} f_{k}(s) p^{b}(s) d s .
\end{array}
$$

This implies that $z(i)-z(i-1)(i=1,2, \ldots, k)$ are independent and identically distributed, and thus $z(n), n=0,1,2, \ldots$, is a random walk. This completes the proof of Lemma 4.

LEMMA 5.

$$
\lim _{\substack{h \rightarrow 0 \\ x \rightarrow b \\(x) / \sqrt{h} \rightarrow-s}} \frac{1}{\sqrt{h}} E_{x}^{h}\left(\phi\left(x_{\tau_{G}}^{h}\right)\right)=E_{-s}^{b}\left(z\left(\tau_{G}\right)\right)
$$

where $x_{n}^{h}$ is a Markov chain with transition density $p_{h}(x, y)$ and $z(n)$ is a random walk with transition density $p^{b}(s)$.

REMARK. The 


$$
\lim _{h \rightarrow 0} \frac{1}{\sqrt{h}} E_{x}^{h}\left(\phi\left(x_{\tau_{G}}^{h}\right)\right)
$$

is nonuniform near the boundary of $G$; thus great care must be taken in how we let $h \rightarrow 0$ and $x \rightarrow b$. Above we let $h \rightarrow 0$ and $x \rightarrow b$ in such a way that

$$
\phi(x) / \sqrt{h} \rightarrow-s
$$

then in order to evaluate

$$
\lim _{x \rightarrow b} \lim _{h \rightarrow 0} \frac{1}{\sqrt{h}} E_{x}^{h}\left(\phi\left(x_{\tau_{G}}^{h}\right)\right)
$$

we shall let $s \rightarrow \infty$. $E_{-s}^{b}\left(z\left(\tau_{G}\right)\right)$ is the expected overshoot in the normal direction over the boundary at $x=b$ of the random walk $z(n)$ starting at $-s$.

Proof. We note that

$$
z_{h}\left(\tau_{G}\right)=(1 / \sqrt{h}) \phi\left(x_{\tau_{G}}^{h}\right)
$$

thus

$$
z_{h}(0)=\phi(x) / \sqrt{h}
$$

and thus

$$
(1 / \sqrt{h}) E_{x}^{h}\left(\phi\left(x_{\tau_{G}}^{h}\right)\right)=E_{\phi(x) / \sqrt{h}}\left(z_{h}\left(\tau_{G}\right)\right) .
$$

By Lemma 4 the finite dimensional distributions of the stochastic process $z_{h}(n)(h=0,1,2, \ldots)$ converge weakly to the finite dimensional distributions of the random walk $z(n)(n=0,1,2, \ldots)$. Since $\tau_{G}=\inf _{n}\{n$ : $\left.z_{h}(n)>0\right\}$ is finite almost surely, it follows by a standard weak convergence argument that

$$
\lim _{\substack{h \rightarrow 0 \\ x \rightarrow b \\ \phi(x) / \sqrt{h} \rightarrow-s}} E_{\phi(x) / \sqrt{h}}^{h} z_{h}\left(\tau_{G}\right)=E_{-s}^{b}\left(z\left(\tau_{G}\right)\right) .
$$

This completes the proof of Lemma 5.

REMARK. Assuming that $p_{0}^{b}(z)$ in Lemma 3 is such that $\iint z p_{0}^{b}(z) d z=0$, then the random walk $z(n)$ will be persistent and by the Renewal Theorem $\lim _{s \rightarrow \infty} E_{-s}^{b} z\left(\tau_{G}\right)$ exists; it is shown in [3] that for a persistent, nonlattice random walk the limiting exit distribution exists.

We shall assume:

$$
\lim _{s \rightarrow \infty} E_{-s}^{b} z\left(\tau_{G}\right) \text { exists uniformly in } b \in \partial G .
$$

In (2.1) we are assuming that if two boundary points are close then so are the expected overshoots, in the normal directions to the boundary at these points, of the random walk $z(n)$. For this it is enough to assume sufficient smoothness of the boundary and the transition density.

Denote $E_{-s}^{b}\left(z\left(\tau_{G}\right)\right)$ by $V_{s}(b)$ and $\lim _{s \rightarrow \infty} V_{s}(b)$ by $V(b)$. Our next objective 
is to show that $\lim _{h \rightarrow 0} \bar{u}_{1}^{h}(x)=\bar{u}_{1}(x)$ exists, $L \bar{u}_{1}=0$, and $\bar{u}_{1}(x)$ takes on the boundary value $W(b) V(b)$ at $b \in \partial G$. To this end we first prove the following

LEMMA 6. Let $P_{n}$ be a sequence of probability measures on a measurable space $(X, \mathscr{F})$ converging weakly to a probability measure $P$, where $X$ is a complete separable metric space and $\mathcal{F}$ its Borel field. Let $C$ be a closed set in $\mathcal{F}$ such that $P(C)=1$; let $f_{n}(x)$ be a sequence of measurable functions on $X$ and $f(x) a$ measurable function on $X$. Furthermore assume

(1) $\left|f_{n}(x)\right| \leqslant K$ for all $n$, and

(2) $\lim _{n \rightarrow \infty ; x_{n} \rightarrow x \in C}\left|f_{n}\left(x_{n}\right)-f(x)\right| \leqslant \delta$; then

$$
\varlimsup_{n \rightarrow \infty}\left|\int_{X} f_{n}(x) d P_{n}(x)-\int_{X} f(x) d P(x)\right|<\delta .
$$

Proof. Suppose the conclusion is not true; then there exist a subsequence $f_{n}(x)$ and $P_{n}$ such that

$$
\varlimsup_{n_{j}}\left|\int_{X} f_{n_{j}}(x) d P_{n_{j}}-\int_{X} f(x) d P(x)\right|=\delta^{\prime}>\delta .
$$

But $P_{n}$ converges weakly to $P$; thus by a theorem of Skorohod there exist a probability space $(\Omega, \Sigma, \Pi)$ and random variables $x_{n}(\omega), x(\omega)$ such that $\Pi\left(x_{n}^{-1}(A)\right)=P_{n j}(A)$, and $\Pi\left(x^{-1}(A)\right)=P(A)$ for all $A \in \mathscr{F} ;$ further, $x_{n}(\omega)$ converges in probability to $x(\omega)$, and there exists a further subsequence $x_{n_{j k}}(\omega)$ which converges to $x(\omega)$, almost surely. Thus by assumption (2)

$$
\varlimsup_{n \rightarrow \infty}\left|f_{n_{j_{k}}}\left(x_{n_{j_{k}}}(\omega)\right)-f(x(\omega))\right|<\delta
$$

a.e., and by the Bounded Convergence Theorem

$$
\varlimsup_{n_{j k}}\left|\int_{\Omega} f_{n_{j k}}\left(x_{n_{j k}}(\omega)\right) d \Pi(\omega)-\int_{\Omega} f(x(\omega)) d \Pi(\omega)\right|<\delta .
$$

But this contradicts the assumption that

$$
\varlimsup_{n_{j}}\left|\int_{X} f_{n_{j}}(x) d P_{n_{j}}(x)-\int_{X} f(x) d P(x)\right|=\delta^{\prime}>\delta .
$$

This completes the proof of Lemma 6.

LEMMA 7. $\lim _{h \rightarrow 0} \bar{u}_{1}^{h}(x)=\bar{u}_{1}(x)$ exists; L $\bar{u}_{1}(x)=0$ for $x \in G$ and $\bar{u}_{1}(b)=$ $W(b) V(b), b \in \partial G$.

Proor. Let $\tau_{s, h}$ be the first exit time of a Markov chain $\left\{x_{n}^{h}\right\}$ with transition density $p_{h}(x, y)$ from $\left\{x: \phi(x)<-s h^{1 / 2}\right\}, s>k$, where $k h^{1 / 2}$ is the uniform bound on the jumps of $\phi\left(x_{n}^{h}\right)$. Then by assumption (2.1), Lemma 2 and Lemma 5 we have 


$$
\lim _{\substack{h \rightarrow 0 \\ x \rightarrow b \\ \phi(x) / \sqrt{h} \rightarrow s^{\prime}}}\left|\bar{u}_{1}^{h}(x)-W(b) V_{s^{\prime}}(b)\right|=0,
$$

where $V_{s^{\prime}}(b)=E_{-s^{\prime}}^{b}\left(z\left(\tau_{G}\right)\right)$. From this it follows that for every $b \in \partial G$,

$$
\begin{aligned}
\lim _{\substack{h \rightarrow 0 \\
x \rightarrow b \\
-s \sqrt{h}<\phi(x)<-s \sqrt{h}+c \sqrt{h}}}\left|\bar{u}_{1}^{h}(x)-W(b) V(b)\right| \\
\quad<\sup _{s-c<s^{\prime}<s}\left|W(b) V_{s^{\prime}}(b)-W(b) V(b)\right| \\
\quad \sup _{b}|W(b)| \sup _{s-c<s^{\prime}<s} \sup _{b}\left|V_{s^{\prime}}(b)-V(b)\right| \\
\quad=\sup _{b}|W(b)| \mathcal{E}(s-c)
\end{aligned}
$$

where $\delta(s)=\sup _{s^{\prime}>s} \sup _{b}\left|V_{s^{\prime}}(b)-V(b)\right|$. By our assumption (2.1) $\mathcal{E}(s) \rightarrow 0$ as $s \rightarrow \infty$. Now by the, strong Markov property

$$
\begin{aligned}
\bar{u}_{1}^{h}(x) & =E_{x}^{h} \bar{u}_{1}\left(x_{\tau_{s, h}}^{h}\right)=\iint_{D} \bar{u}_{1}^{h}(x) H_{h}^{s}(x, d y), \\
D & =\left\{x:-s h^{1 / 2} \leqslant \phi(x) \leqslant-s h^{1 / 2}+c h^{1 / 2}\right\},
\end{aligned}
$$

where $H_{h}^{s}(x, d y)$ is the exit distribution from $\left\{s:-s h^{1 / 2}>\phi(x)\right\}$. It is well known that for regular boundary points $b \in \partial G$,

$$
\lim _{\substack{h \rightarrow 0 \\ x \rightarrow b}} H_{h}^{s}(x, d y)=H(x, d b),
$$

where $H(x, d b)$ is the harmonic measure on $\partial G$. Setting $C=\partial G, f_{n}=\bar{u}_{1}^{h}$, $f=\bar{u}, P_{n}=H_{h}^{s}, P=H$ and $\delta(s)=\delta$ in Lemma 6 , and noting that all the assumptions of Lemma 6 are met, we can conclude by Lemma 6 that

$$
\begin{aligned}
\varlimsup_{h \rightarrow 0}\left|\bar{u}_{1}^{h}(x)-\bar{u}_{1}(x)\right| \\
\quad=\varlimsup_{h \rightarrow 0}\left|\iint_{D} \bar{u}_{1}^{h}(x) H_{h}^{s}(x, d y)-\int_{\partial G} W(b) V(b) H(x, d b)\right| \\
\quad<\delta(s-c) .
\end{aligned}
$$

But $\bar{u}_{1}^{h}(x)$ and $\bar{u}_{1}(x)$ are independent of $s$, and $\delta(s-c) \rightarrow 0$ as $s \rightarrow \infty$. This completes the proof of Lemma 7 .

Recalling that $u_{1}(x)=\hat{u}_{1}(x)+\bar{u}_{1}(x)$ where $\hat{u}_{1}(x)$ satisfies the Poisson equation, $L \hat{u}_{1}=-g_{1}$, with zero boundary condition and $\bar{u}_{1}(x)$ is harmonic for $L$ inside $G$ with the boundary values given by Lemma 7. We can now summarize our results by the following

THEOREM. Let 


$$
\begin{aligned}
A & =\{x: \phi(x) \leqslant d<0\}, \\
V(b) & =\lim _{\substack{h \rightarrow 0 \\
x \rightarrow b \\
\phi(x) / \sqrt{h} \rightarrow \infty}} \frac{1}{\sqrt{h}} E_{x}^{h} \phi\left(x_{\tau_{c}}^{h}\right)
\end{aligned}
$$

and

$$
W(x)=\frac{\langle\nabla(F(x)-U(x)), \nabla \phi(x)\rangle}{\|\nabla \phi(x)\|^{2}}
$$

then

$$
\lim _{h \rightarrow 0} \sup _{x \in A}\left|\frac{u_{h}(x)-u(x)}{\sqrt{h}}-u_{1}(x)\right|=0,
$$

where $u_{1}(x)$ is the solution to $L u_{1}(x)=-g_{1}(x)$ with boundary values $u_{1}(b)=$ $W(b) V(b), b \in \partial G$.

Acknowledgement. The contents of this paper are a part of the author's Ph.D. thesis, written under the direction of Professor S.R.S. Varadhan. The author wishes to express his heartfelt gratitude to Professor Varadhan for his guidance and encouragement.

\section{REFERENCES}

1. L. Bers, F. John and M. Schecter, Partial differential equations, Lectures in Appl. Math., Vol. III, Wiley, New York, 1964. MR 29 \#346.

2. E. Dynkin, Infinitesimal operators of Markov processes, English transl., Theor. Probability Appl. 1 (1956), 34-54. MR 19, 691.

3. W. Feller, An introduction to probability theory and its applications, Vol. 2, 2nd ed., Wiley, New York, 1971. MR 42 \#5292.

4. G. Forsythe and W. Wasow, Finite-difference methods for partial differential equations, Wiley, New York, 1960. MR 23 \#B3156.

Department of Mathematics, long Island Universtty, Brookiyn Center, Brooklyn, NEW YORK 11201 